\title{
Thermal-Aware Design of 3D ICs with Inter-Tier Liquid Cooling
}

\author{
David Atienza $\ddagger$ \\ ${ }^{\ddagger}$ Embedded Systems Laboratory (ESL), Ecole Polytechnique Fédérale de Lausanne (EPFL), Switzerland. \\ EPFL-STI-IEL-ESL, Station 11; 1015 Lausanne, Switzerland; E-mail: david.atienza@epfl.ch
}

Advances in manufacturing technologies are enabling the development of 3D multi-processor ICs, but heat in 3D stacking can lead to degraded performance due to higher power density, which derives from the placement of computational units and storage components on top of each other [1]. Since it is more difficult to remove the heat from 3D ICs, cooling can become the main limiting factor for future 3D multiprocessor system-on-chip (MPSoCs). This paper presents a novel thermal-aware design paradigm for 3D ICs, which includes thermal modeling as a fundamental step to design 3D with inter-tier liquid cooling as well as system-level dynamic thermal management to tune the coolant flow rate to achieve energy-efficient thermally-balanced 3D MPSoCs.

Thermal modeling of 3D MPSoCs with liquid cooling Inter-tier liquid cooling is a potential solution to address the high temperatures in 3D MPSoCs, due to the higher heat removal capability of liquids in comparison to air [3]. This technology involves injecting water through micro-channels between the tiers of a 3D stack using a pump to remove the heat. Fig. 1 shows layouts of 3D MPSoCs developed with this technology, which consists of two or more stacked layers (with cores, L2 caches, crossbar, memory control units, etc.), and water microchannels [1] are distributed uniformly in between the vertical layers. Then, we alter at runtime the liquid flow rate to minimize the pump energy consumption, which can represent more than $40 \%$ of total energy consumption of 3D MPSoCs [4]. To this end, in order to characterize the run-time temperature of 3D MPSoCs, we have developed 3D-ICE [3], which is a compact transient thermal model for the thermal simulation of 3D ICs with multiple inter-tier liquid cooling microchannels. 3D-ICE is compatible with existing CAD tools for ICs, and offers significant speed-ups (up to 975x) over typical commercial computational fluid dynamics simulation tools while preserving accuracy (i.e., maximum temperature error of $3.4 \%$ ).

System-Level Thermal Management of 3D MPSoCs Finally, based on 3D-ICE we have developed a new family of global $\mathrm{HW} / \mathrm{SW}$ temperature controllers for energy-efficient 3D MPSoC cooling [4]. These controllers include a thermalaware job scheduler, which balances the temperature across the system to maximize cooling efficiency. Then, they forecast maximum system temperature and proactively set the liquid flow rate. The experiments on 4-layered 3D MPSoCs (Figure 2) show that these controllers, i.e., a liquid cooling controller based on fuzzy logic (LC_fuzzy) or targeting global load balancing (LC_LB), prevent the system to exceed the given threshold temperature $\left(85^{\circ} \mathrm{C}\right)$ while reducing cooling

This research is partially funded by the Nano-Tera RTD project CMOSAIC (ref.123618), financed by the Swiss Confederation and scientifically evaluated by SNSF, and the PRO3D EU FP7-ICT-248776 project.
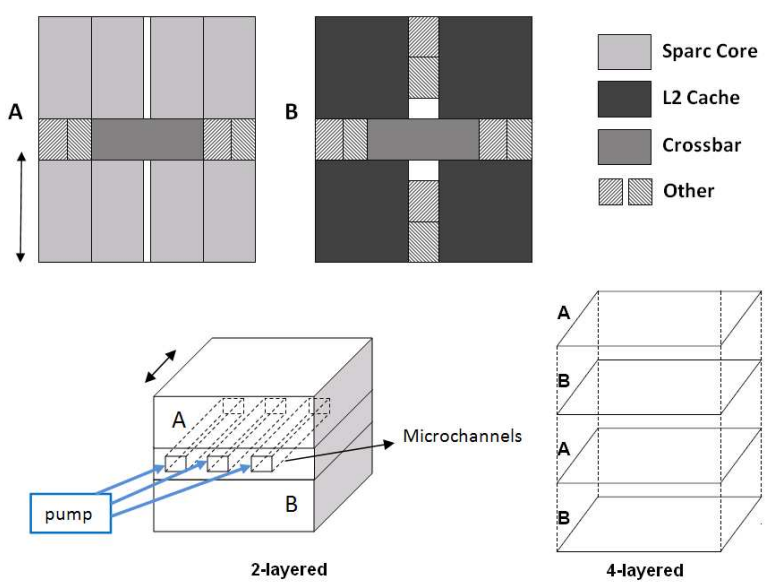

Fig. 1. 3D MPSoCs with inter-tier liquid cooling

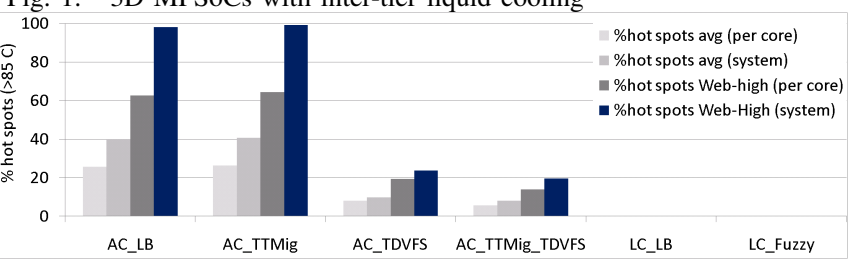

Fig. 2. Percentage of time of hot-spots per core and across the entire die for 4-tier MPSoC designs running different system-level thermal control policies (i.e., average case across all workloads and for Web-high, the hottest benchmark)

energy by up to $50 \%$ with respect to a worst-case flow rate setting. Also, they improve system-level energy by up to $21 \%$ with respect to state-of-the-art thermal controllers using air cooling, i.e., dynamic load balancing (AC_LB), temperaturetriggered task migration (AC_TTMig) or temperature-triggered dynamic frequency and voltage scaling (AC_TDVFS).

\section{Conclusion}

Microchannel-based liquid cooling is a promising technology solution to overcome the thermal challenges of 3D MPSoCs. However, intelligent control of the coolant flow rate is needed to avoid wasted energy consumption for over-cooling the system when the system is under-utilized. Therefore, this paper proposes a novel system-level thermal-aware design as an affective method to achieve thermally-balanced 3D MPSoCs.

\section{References}

[1] T. Brunschwiler, et al., "Interlayer cooling potential in vertically integrated packages", Microsyst. Technol., 2009.

[2] A. K. Coskun, et al., "Dynamic thermal management in 3D multicore architectures", Proc. DATE, 2009.

[3] A. Sridhar, et al., "3D-ICE: Fast compact transient thermal modeling for 3D-ICs with inter-tier liquid cooling", Proc. ICCAD, 2010.

[4] A. K. Coskun, et al., "Energy-efficient variable-flow liquid cooling in 3D stacked architectures", Proc. DATE, 2010. 